\title{
Future therapies: Early trials and basic science
}

\author{
Karl-Erik Andersson, MD, PhD \\ Professor, Institute for Regenerative Medicine, Wake Forest University School of Medicine, Winston-Salem, NC
}

Cite as: Can Urol Assoc J 2013;7(9-10):S179-80. http://dx.doi.org/10.5489/cuaj.1617 Published online October 9, 2013.

\section{Abstract}

Pharmacotherapeutic options for overactive bladder (OAB) include antimuscarinics and the beta3-adrenoceptor agonist mirabegron. Research and development of novel therapeutic options for OAB continues to be an active field. This review summarizes recent research with the existing therapies as well as the most promising agents in development.

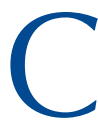

urrent options for the pharmacologic management of overactive bladder $(\mathrm{OAB})$ include antimuscarinic agents and the beta3-adrenoceptor ( $\beta 3$-AR) agonist mirabegron. These therapies can provide symptom relief for many patients with $\mathrm{OAB}$, but there is still considerable room for refining and expanding the therapeutic armamentarium. This brief review summarizes the current status of recent and ongoing research with novel therapeutic approaches, as well as recent evidence on ways to better treat $\mathrm{OAB}$ symptoms using the agents we already have at our disposal.

\section{Antimuscarinic therapy}

There is one new molecule, imidafenacin, that has become available in certain parts of the world. It was designed to be selective to the $\mathrm{M} 1$ and $\mathrm{M} 3$ receptors, with the aim of reducing anticholinergic side effects. ${ }^{1}$ While its efficacy and safety are well documented from randomized clinical trials, ${ }^{1}$ there do not appear to be any obvious advantages over other antimuscarinics. Imidafenacin is not currently available in Canada (or the United States).

Another method that is being explored to reduce the burden of antimuscarinic-related adverse effects is the use of a muscarinic receptor agonist, pilocarpine, in a fixed-dose combination with an antimuscarinic, tolterodine. In a phase II study, this combination was shown to reduce the incidence of dry mouth by $60 \%$ compared to tolterodine alone, while achieving significant improvements of $\mathrm{OAB}$ and urge urinary incontinence symptoms relative to placebo. ${ }^{2}$ Research is ongoing with this combination.

There has also been some interesting basic research completed that may help explain why antimuscarinic therapy fails to con- trol symptoms in many patients. Evidence from an animal model has shown that chronic administration of antimuscarinic agents (oxybutynin or fesoterodine) leads to loss of muscarinic receptor efficiency and induces a shift from muscarinic to purinergic transmission. ${ }^{3}$ Should this prove to be true in humans as well, the implication might be that it could be more beneficial to administer antimuscarinic therapy on an intermittent basis.

\section{Combination therapy}

$\beta 3-A R$ agonists exert their therapeutic effects through stimulation of adenylyl cyclase and activation of potassium $\mathrm{K}+$ channels. The former leads to an increase in cyclic adenosine monophosphate and the latter to hyperpolarization, both of which result in relaxation. The beneficial effects of modulation of these pathways are: inhibition of spontaneous activity, increased bladder compliance (decreased bladder tone during filling), greater distension needed to activate the micturition reflex (increased bladder capacity), and decreased afferent activity, with no effect on voiding contraction (no risk for urinary retention).

These mechanisms are distinct from those of antimuscarinic therapies used to treat $\mathrm{OAB}$. As such, the combination of these two types of medications is being investigated to determine whether concomitant use can result in increased efficacy with an acceptable profile of safety and tolerability. At the 2013 meeting of the American Urological Association (AUA), researchers working with animal models concluded that the "combination of antimuscarinics and $\beta 3$-adrenoceptor agonists can result in increased efficacy and potency and supports the hypothesis that combining these compound classes in the clinic could have beneficial effects in treating urinary bladder dysfunction." 4

Indeed, at the same congress, researchers presented the results of a phase II clinical study (the Symphony study) evaluating the combination of solifenacin and mirabegron in 1307 patients with $\mathrm{OAB} .{ }^{5}$ The subjects were randomized to receive one of six combinations: mirabegron 25 or $50 \mathrm{mg}$ in combination with solifenacin 2.5, 5 or $10 \mathrm{mg}$; monotherapy with mirabegron or solifenacin (at each of the same doses studied in the combinations); or placebo. The study duration was 12 weeks. The primary efficacy variable was change in mean volume voided (MVV) per micturition; secondary variables included change in micturition frequency (MF) and 
incontinence episode frequency per 24 hours. The investigators reported that mirabegron combination therapy with solifenacin (the latter at a dose of $>5 \mathrm{mg}$ ) demonstrated greater efficacy than solifenacin $5 \mathrm{mg}$ alone on MVV and MF. ${ }^{5}$ The enhanced efficacy with the combination is of a magnitude that is probably similar to the enhanced efficacy one might expect from uptitrating the dose of the antimuscarinic. However, the combination is not associated with the adverse effects one would expect to encounter with higher doses of antimuscarinics. In this study, all six combinations appeared to be well-tolerated and there appeared to be no safety concern or significant increase in adverse effects with the combination treatment compared with either monotherapy. ${ }^{5}$

\section{Investigational therapies with other mechanisms of action}

There are a number of different therapeutic pathways being explored in preclinical studies that have potential utility for the treatment of OAB (Table 1). These include purinergic receptor antagonism, TRP channel antagonism, cannabinoid receptor agonism, melatonin modulation and apoptosis-inducing agents.

To date, the only agents that have made it from the laboratory into clinical trials for urinary tract symptoms are the apoptosisinducing agents NX-1207 and PRX302, which are administered as intraprostatic injections for men with benign prostatic hyperplasia. NX-1207 has been associated with prostate volume reduction, as well as with short- and long-term symptomatic improvement in phase II studies, ${ }^{6}$ and is currently being studied in phase III clinical trials.

In a phase IIb trial involving 92 patients with International Prostate Symptom Score (IPSS) scores of 15 or higher at baseline, treatment with PRX302 was associated with a 9-point reduction in IPSS and an increase of $3 \mathrm{~mL}$ per second in peak urine flow (both changes statistically significant). ${ }^{7}$

\section{Conclusions}

Research and development of pharmacotherapies for the treatment of urinary tract symptoms is an active and dynamic field. In addition to identifying and exploring a number of new treatment pathways, researchers are also continuing to expand the knowledge base about the way currently available therapies work alone and in combination. Whether or not any of the strategies currently being investigated end up supplanting antimuscarinic therapy as the standard first-line therapy remains to be seen.

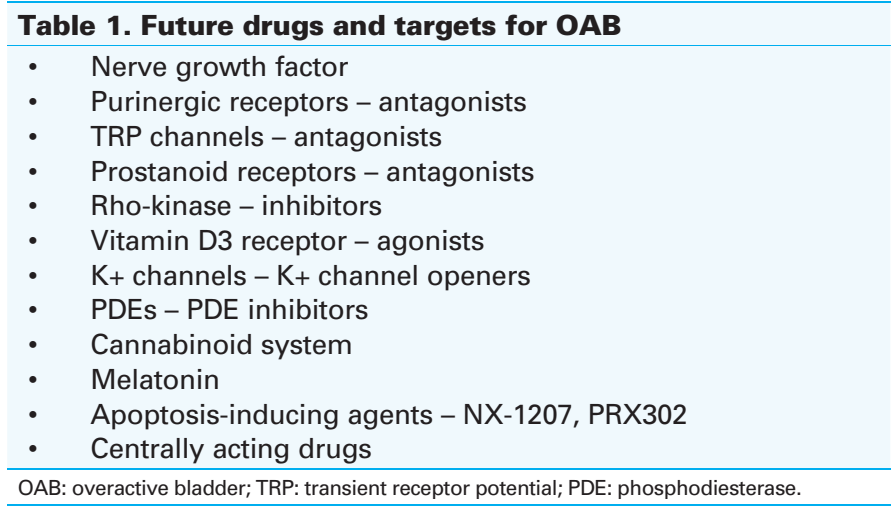

Competing interests: This article is part of a CUAJ supplement sponsored by Astellas Pharma Canada, Inc. Dr. Andersson has been a paid consultant with Allergan, Astellas, Ferring, and Ono.

\section{References}

1. Maggiore UL, Scala C, Venturini PL, et al. Imidafenacin for the treatment of overactive bladder. Expert Opin Pharmacother 2013;14:1383-97. http://dx.doi.org/10.1517/14656566.2013.796930

2. Dmochowski R, Duchin K, Tremblay T, et al. Tolenix ${ }^{\text {TM }}$ (THVD-201), a novel combination of muscarinic agonist (tolterodine) and muscarinic agonist (pilocarpine), is efficacious in $\mathrm{ABB}$ with less dry mouth compared to tolterodine alone [EAU abstract \#402]. Eur Urol Suppl 2013;12:e402-e403. http:// dx.doi.org/10.1016/S1569-9056(13)60887-8

3. Uvin P, Boudes $M$, Franken J, et al. Why anticholinergics fail: oxybutynin and fesoterodine induce a shift from muscarinergic to purinergic transmission in the rat bladder [EAU abstract \#441]. Eur Urol Suppl 2013;12:e441. http://dx.doi.org/10.1016/S1569-9056(13)60925-2

4. Rekik M, Rouget C, Palea $S$, et al. Effects of combining antimuscarinics and $\beta 3$-adrenoceptor agonists on contractions induced by electrical field stimulation of rat isolated urinary bladder strips [AUA abstract 283]. J Urol 2013;189:e115-e116. http://dx.doi.org/10.1016/i.juro.2013.02.1667

5. Abrams $P$, Kelleher C, Staskin D, et al. Combination treatment with mirabegron and solifenacin in patients with overactive bladder (OAB) - efficacy results from a phase 2 study (Symphony) [AUA abstract 1958]. J Urol 2013;189:e803. http://dx.doi.org/10.1016/i.juro.2013.02.2377

6. Shore N. NX-1207: a novel investigational drug for the treatment of benign prostatic hyperplasia. Expert Opin Investig Drugs 2010;19:305-10. http://dx.doi.org/10.1517/13543780903555196

7. Elhilali MM, Pommerville P, Yocum RC, et al. Prospective, randomized, double-blind, vehicle controlled, multicenter phase llb clinical trial of the pore forming protein PRX302 for targeted treatment of symptomatic benign prostatic hyperplasia. J Urol 2013;189:1421-6. http://dx.doi.org/10.1016/i.juro.2012.11.003

Correspondence: Dr. Karl-Erik Andersson, Institute for Regenerative Medicine, Wake Forest University School of Medicine, Medical Center Boulevard, Winston Salem, NC, 27157; keanders@wakehealth.edu 